\title{
COIMPARATIVE EVALUATION OF LMA CTRACH VERSUS LMA FASTRACH FOR TRACHEAL INTUBATION
}

KEY WORDS: Intubation, Laryngeal Mask Airway, General Anaesthesia, Airway

\section{Manish Kumar Tiwary*}

Raminder Sehgal

Subhash Gupta

\section{Jayashree Sood}

Department of Anaesthesia, Sir Ganga Ram Hospital, Rajinder Nagar, New Delhi-110060, India. *Corresponding Author

Department of Anaesthesia, Sir Ganga Ram Hospital, Rajinder Nagar, New Delhi-110060, India.

Department of Anaesthesia, Sir Ganga Ram Hospital, Rajinder Nagar, New Delhi-1 10060, India.

Department of Anaesthesia, Sir Ganga Ram Hospital, Rajinder Nagar, New Delhi-1 10060, India.

Introduction: The intubating laryngeal mask airway (ILMA) or LMA Fastrach ${ }^{\mathrm{TM}}$ was invented as a ventilatory device, and as a conduit for blind tracheal intubation. However, intubation through LMA Fastrach ${ }^{\mathrm{TM}}$ requires multiple attempts. LMA $\mathrm{CTrach}^{\mathrm{TM}}$ is a modification of LMA Fastrach ${ }^{\mathrm{TM}}$ and allows intubation under direct vision via an integrated fibreoptic bundle and a detachable liquid crystal display viewer. This study was undertaken to compare the first attempt success rate of tracheal intubation through the CTrach laryngeal mask and LMA Fastrach ${ }^{\mathrm{TM}}$. Methods and Materials: This prospective randomized study was conducted on 100 adults, ASA physical status I and II patients of either sex, scheduled to undergo general anaesthesia requiring tracheal intubation for elective surgery. The patients were randomly allocated to two groups of 50 patients each, to be intubated via CTrach (GROUP CT, $n=50$ ) or ILMA (GROUP FT, n=50). The first-attempt and overall success rates of tracheal intubation, the times taken and incidence of pharyngo-laryngeal morbidity, were recorded.

Results: Tracheal intubation was successful on the first attempt in $96 \%$ of patients with the LMA CTrach ${ }^{\mathrm{TM}}$ and $84 \%$ of patients with the LMA Fastrach ${ }^{\mathrm{TM}}(p=0.045)$. The success rates within three attempts were $100 \%$ in both groups $(p=0.124)$. The mean time taken for the complete tracheal intubation process was $109.6 \pm 38.679$ seconds with the LMA CTrach $^{\mathrm{TM}}$ and $118.68 \pm 22.342$ seconds with the LMA Fastrach ${ }^{\mathrm{TM}}(p=0.154)$.

Conclusion: To conclude, both airway devices are comparable and efficacious and the overall success of tracheal intubation was $100 \%$ in both groups.

\section{INTRODUCTION:}

Despite recent developments in equipment and techniques, anaesthesiologists continue to face difficulty in tracheal intubation in $13 \%$ cases undergoing general anaesthesia. ${ }^{1}$ Airway mismanagement continues to account for the majority of serious morbidity and mortality related to anaesthesia. The LMA has a role in the management of difficult airway, both as a primary airway and as a guide to intubation. It has been given a prominent place in the ASA difficult airway algorithm at five places. ${ }^{3}$ It is one of the three non-surgical techniques recommended for use in "cannot intubate cannot ventilate" scenario. It has also been used as a conduit for tracheal intubation, but the success rate of blind intubation through standard CLMA ranges from $30 \%$ to $90 \% .{ }^{4}$ Moreover, the largest size endotracheal tube which can be negotiated through size 3 or $4 \mathrm{LMA}$ is $6 \mathrm{~mm}$ internal diameter (ID). Also, it does not protect the airway from aspiration as effectively as the endotracheal tube (ETT).

The intubating laryngeal mask airway (ILMA) or LMA Fastrach ${ }^{\mathrm{TM}}$ was invented in 1997 as a further development of the classic laryngeal mask airway. ${ }^{5,6}$ It was designed for use as a ventilatory device, and in conjunction with a dedicated ETT, as a conduit for blind tracheal intubation while maintaining ventilatory properties of the classic LMA (CLMA). The main reason that prompted its introduction was that it overcomes the length limitations of tracheal tube. Moreover, it does not require intraoral digital manipulation or head and neck manipulation for placement and the epiglottis is elevated from the intubating path. It permits single handed intubation without moving head from neutral position and has become a valuable tool in management of anticipated and unanticipated difficult airway.

LMA guided intubation has always been a great challenge in airway management of unanticipated difficult airway with a need to secure the airway at the earliest. Starting from the days of LMA guided intubation through classic LMA to the introduction of ILMA, it has been a long journey but still there remains more scope to re-evaluate and improve on it. The LMA CTrach ${ }^{\mathrm{TM}}$ is a new device which was made commercially available in $2005 .^{9,10}$ It is a modification of LMA Fastrach ${ }^{\mathrm{TM}}$ and consists of LMA CTrach ${ }^{\mathrm{TM}}$ airway conduit with an integrated fibreoptic system and a detachable viewer. A lens situated behind the epiglottic elevator captures an image from in front of the mask aperture, which is transmitted to a detachable digital screen, thus providing a view of the larynx. It is reported to facilitate placement of endotracheal tube into the larynx. ${ }^{11}$ This study was undertaken to compare the first attempt success rate of tracheal intubation through the CTrach laryngeal mask and LMA Fastrach ${ }^{\mathrm{TM}}$. The secondary outcomes were to determine number of attempts taken to intubate, time taken for tracheal intubation, incidence of pharyngo-laryngeal morbidity (with respect to Blood on airway device, sore throat lip, tongue and dental trauma).

\section{MATERIALS AND METHODS:}

After obtaining approval from the hospital ethics committee and written informed consent from the patients, this prospective randomized study was conducted on 100 adults, American Society of Anesthesiologists'-physical status (ASAPS) I and II patients of either sex, scheduled to undergo general anaesthesia requiring tracheal intubation for elective obstetric and gynaecological surgery. Fifty patients were randomly allocated by computer generated numbers to one of the following two groups: group CT $(n=50)$, tracheal intubation using LMA CTrach ${ }^{\mathrm{TM}}$, group FT $(n=50)$ : Tracheal intubation using LMA Fastrach ${ }^{\mathrm{TM}}$. We excluded patients with ASA grade III and IV, body mass index (BMI)more than 35 $\mathrm{kg} / \mathrm{m}^{2}$, History of gastro-oesophageal reflux disease, Gastric tumour, Pregnancy, Patients fasting less than six hours. A detailed pre-anaesthetic examination comprising of history, general physical and systemic examination was done for all patients. Airway was evaluated by modified Mallampatti 
classification. All patients were kept fasting for 6 hours prior to surgery.

Anaesthesia technique was standardized for all patients. On arrival in the operation theatre, standard anaesthesia monitoring comprising of pulse oximeter, non-invasive blood pressure and electrocardiography were attached. All patients received ranitidine hydrochloride $\mathrm{lmg} / \mathrm{kg}$, metoclopramide $0.2 \mathrm{mg} / \mathrm{kg}$ and glycopyrrolate $0.04 \mathrm{mg} / \mathrm{kg}$ intravenously slowly over 30 seconds. Patients were positioned supine with a silicone donut $5 \mathrm{~cm}$ in height placed under the head. All patients received midazolam $0.01-0.05 \mathrm{mg} / \mathrm{kg}$ and fentanyl citrate $1 \mathrm{~g} / \mathrm{kg}$. After preoxygenation for 3 minutes, anaesthesia was induced with propofol $1.5-2 \mathrm{mg} / \mathrm{kg}$. After assessing bag and mask ventilation, muscle relaxation was achieved with $0.5-\mathrm{mg} / \mathrm{kg}$ atracurium besylate. The patient's lungs were manually ventilated for 3 minutes by a face mask of appropriate size. An initial laryngoscopy was done to assess the best Cormack and Lehane grade using Macintosh laryngoscope by an anaesthesiologist not involved in study.

LMA Fastrach ${ }^{\mathrm{TM}}$, LMA CTrach ${ }^{\mathrm{TM}}$ and reusable, flexible, cuffed, wire-reinforced LMA Fastrach ${ }^{\mathrm{TM}}$ tracheal tube were selected as per manufacturer's recommendations with size $3 \mathrm{LMA}$ and $7.0 \mathrm{~mm}$ internal diameter (ID) ETT for the patients with body weight below $50 \mathrm{~kg}$, size $4 \mathrm{LMA}$ and $7.5 \mathrm{~mm}$ ID ETT for the patient with body weight $50-70 \mathrm{~kg}$ and size $5 \mathrm{LMA}$ and $8.0 \mathrm{~mm}$ ETT for the patients with body weight more than 70kg. LMA CTrach $^{\text {TM }}$ or LMA Fastrach ${ }^{\mathrm{TM}}$ were inserted as per the group allocation with patient's head in neutral position and intubation was performed with minimum neck movement. The patient's lungs were ventilated in between attempts to ensure oxygen saturation $>95 \%$ at all times. In the LMA Fastrach we inserted the LMA Fastrach ${ }^{\mathrm{TM}}$, inflated the cuff, and checked our ability to ventilate the lungs. If ventilation was difficult, we first applied the "up-down manoeuvre" by withdrawing the LMA Fastrach ${ }^{\mathrm{TM}}$ by $6 \mathrm{~cm}$ and reinserting it, with the cuff still inflated. If this failed, we partially withdrew the LMA Fastrach ${ }^{\mathrm{TM}}$, and if this also failed, we completely removed and reinserted the LMA Fastrach ${ }^{\mathrm{TM}}$. The same sequence was followed by all investigators. In the LMA CTrach ${ }^{\mathrm{TM}}$ group, we also optimized ventilation after insertion of the LMA CTrach ${ }^{\mathrm{TM}}$ using the same sequence of manoeuvres as with the $L M A$ Fastrach ${ }^{T M}$, always starting with the up-down manoeuvre. We then attached the viewer and adjusted the LMA CTrach ${ }^{\mathrm{TM}}$ to obtain a full view of the glottis.

If intubation was not achieved by LMA CTrach ${ }^{\mathrm{TM}}$ or LMA Fastrach $^{\mathrm{TM}}$ in 3 attempts, tracheal intubation was performed using Macintosh blade laryngoscope. Correct placement of the endotracheal tube was confirmed by capnograph and LMA CTrach ${ }^{\mathrm{TM}}$ and LMA Fastrach ${ }^{\mathrm{TM}}$ removed over the tube with help of the stabilizing rod. Anaesthesia was maintained with $2-2.5 \%$ sevoflurane and $66 \%$ nitrous oxide in oxygen. Muscle relaxation was achieved with intermittent bolus doses of injection atracurium. Time taken for tracheal intubation and the number of attempts in the two groups were recorded.Time taken for tracheal intubation was taken from the introduction of LMA CTrach ${ }^{\mathrm{TM}}$ or LMA Fastrach ${ }^{\mathrm{TM}}$ into mouth till the confirmation of tracheal tube placement by end tidal carbon dioxide $\left(\mathrm{ETCO}_{2}\right)$ trace.

Results were statistically analysed with SPSS version 17.0 software (Chicago, IL, USA). The categorical data was analysed with Chi-Square tests, ordinal data with MannWhitney $\mathrm{U}$ tests, and continuous data with unpaired $t$ tests. Continuous data was presented as mean SD and ordinal data as median and interquartile range. Categorical data was presented as number of patients and percentage. $p$ value $<$ 0.05 was taken as significant.

\section{RESULTS:}

During the 24-month period (November 2008 to October 2010), 100 adult, ASA I and II patients of either sex, scheduled to undergo general anaesthesia requiring tracheal intubation for elective surgery were included in the study. Demographic data for the two study groups were similar (table 1). Tracheal intubation was successful on the first attempt in 42 of 50 patients (84\%) in the LMA Fastrach ${ }^{\mathrm{TM}}$ group compared with 48 of 50 patients $(96 \%)$ in the LMA CTrach ${ }^{\text {TM }}$ group $(p<0.05)$. The overall success rates of tracheal intubation were $100 \%$ in both groups. One patient in the LMA Fastrach ${ }^{\mathrm{TM}}$ group required tracheal intubation with three attempts. The time taken for tracheal intubation and the pharyngolaryngeal morbidity was comparable between the two groups $(p>0.05)$. Table 2 shows the comparison of LMA and insertion related characteristics.

Table 1: Comparison of demographic data

\begin{tabular}{|l|l|l|l|}
\hline Variable & Group CT & Group FT & p-value \\
\hline Age $(\mathrm{yrs})$ & $38.68+11.91$ & $40.22+10.93$ & 0.502 \\
\hline Gender $(\mathrm{M} / \mathrm{F})$ & $2 / 48$ & $0 / 50$ & 0.495 \\
\hline Weight $(\mathrm{kg})$ & $60.90+5.411$ & $61.40+3.44$ & 0.583 \\
\hline Height $(\mathrm{cm})$ & $155+3.511$ & $155.08+2.86$ & 0.583 \\
\hline ASA-PS (I/II) & $31 / 19$ & $30 / 20$ & 0.838 \\
\hline MPC (I/II) & $36 / 14$ & $38 / 12$ & 0.648 \\
\hline Dentition(N/L/M) & $47 / 1 / 2$ & $44 / 1 / 5$ & \\
\hline CL grade (1/2/3/4) & $37 / 13 / 0 / 0$ & $38 / 12 / 0 / 0$ & 0.817 \\
\hline
\end{tabular}

Table 2: Comparison of size of LMA, attempts, intubation characteristics.

\begin{tabular}{|l|l|l|l|}
\hline Variable & $\begin{array}{l}\text { Group } \\
\text { CT }\end{array}$ & Group FT & $\begin{array}{l}\mathrm{p}- \\
\text { value }\end{array}$ \\
\hline LMA size & $2 / 47 / 1$ & $0 / 50 / 0$ & 0.213 \\
\hline $\begin{array}{l}\text { Number of attempts } \\
\text { Success of intubation in first } \\
\text { attempt } \\
\begin{array}{l}\text { Success of intubation within } 3 \\
\text { attempts (I/II/III/FAIL) }\end{array}\end{array}$ & $48 / 50$ & $42 / 50$ & 0.045 \\
\hline Time taken for intubation (sec) & $\begin{array}{l}109.6+38 \\
679\end{array}$ & $\begin{array}{l}118.68+22 \\
.342\end{array}$ & 0.154 \\
\hline $\begin{array}{l}\text { Optimum manipulation } \\
\text { (NIL/UD/PW/CW) }\end{array}$ & $29 / 13 / 6 / 2$ & $22 / 14 / 6 / 8$ & 0.352 \\
\hline Ease of intubation(I/II/III/IV) & $29 / 19 / 2 / 0$ & $23 / 19 / 8 / 0$ & 0.248 \\
\hline $\begin{array}{l}\text { Pharyngo-laryngeal morbidity } \\
\text { (Blood on airway) } \\
\text { (Sore Throat) }\end{array}$ & $1 / 50$ & $2 / 50$ & 1.00 \\
$2 / 50$ & $4 / 50$ & 0.678 \\
\hline
\end{tabular}

\section{DISCUSSION}

The present study demonstrates that both LMA CTrach ${ }^{\mathrm{TM}}$ and LMA Fastrach ${ }^{\mathrm{TM}}$ are useful airway devices as conduits for intubation of the trachea. All patients recruited for the present study could be successfully intubated with these devices within 3 attempts.

The LMA Fastrach ${ }^{\mathrm{TM}}$, a prototype of CLMA, was introduced in 1997 for blind tracheal intubation. ${ }^{5,6}$ It has an established role in airway management, enabling ventilation and providing a conduit for tracheal intubation. It is also recommended in situations where both mask ventilation and conventional tracheal intubation are difficult. ${ }^{7}$ However, despite corrective manoeuvres and multiple attempts, blind tracheal intubation with LMA Fastrach ${ }^{\text {TM }}$ frequently fails. The success rate of tracheal intubation through LMA Fastrach ${ }^{\mathrm{TM}}$ on first attempt is reported to be between $80-93 \%$ in different studies. ${ }^{12,13}$ Baskett and co-workers evaluated LMA Fastrach ${ }^{\mathrm{TM}}$ in 500 surgical patients and reported $80 \%$ success rate of tracheal intubation on the first attempt. Kapila and colleagues evaluated intubating laryngeal mask airway (LMA Fastrach ${ }^{\mathrm{TM}}$ ) to facilitate tracheal intubation in 100 fasted patients presenting for elective surgery. They found overall success rate of tracheal intubation to be $93 \%$. Avidan investigated the ability of inexperienced personnel to intubate via direct laryngoscopy and compared this with their ability to intubate via LMA Fastrach ${ }^{\mathrm{TM}} .{ }^{14}$ Success rate of insertion and ventilation via the LMA Fastrach ${ }^{\mathrm{TM}}$ was $98 \%$. 
Archie Brain developed LMA CTrach ${ }^{\mathrm{TM}}$, a new device which was made commercially available in $2005^{9,10}$ It was a modification of LMA Fastrach ${ }^{\mathrm{TM}}$ and consists of LMA CTrach ${ }^{\mathrm{TM}}$ airway conduit with an integrated fibreoptic system and a detachable viewer. A lens situated behind the epiglottic elevator captures an image from in front of the mask aperture, which is transmitted to a detachable digital screen, thus providing a view of the larynx. It is reported to improve the first attempt success rate of tracheal intubation through the CTrach laryngeal mask conduit as compared to blind intubation through LMA Fastrach ${ }^{\mathrm{TM}}$.

Liu and colleagues compared tracheal intubation with LMA Fastrach $^{\mathrm{TM}}$ and LMA CTrach ${ }^{\mathrm{TM}}$ system in 271 adult patients requiring intubation. They concluded that the ability to view the glottis and optimize placement of the LMA CTrach ${ }^{\mathrm{TM}}$ under vision enabled a higher first attempt success rate of tracheal intubation being $93.3 \%$ with the LMA CTrach ${ }^{\mathrm{TM}}$ compared to $^{\circ}$ $67.9 \%$ with the LMA Fastrach ${ }^{\mathrm{TM}}{ }^{8}$. Similarly, Goldman and colleagues reported a prospective observational evaluation of 328 uses of the device (LMA CTrach ${ }^{\mathrm{TM}}$ ) at 3 United States centres. First-time and overall intubation success rates were $97 \%(319 / 328)$ and $91 \%(297 / 328)$ respectively. ${ }^{15}$

Dhonneur and colleagues compared tracheal intubation with LMA CTrach ${ }^{\mathrm{TM}}$ and direct laryngoscopy (DL) in morbidly obese patients. ${ }^{16,17}$ They concluded that the LMA CTrach ${ }^{\mathrm{TM}}$ was an efficient airway device for ventilation and tracheal intubation in case of a difficult airway in morbidly obese patients. They observed $100 \%$ success rate of tracheal intubation with both the techniques but forty-nine percent of the patients from the CT group required laryngeal mask manipulation for adequate ventilation and view optimization.

In a study on the use of LMA CTrach ${ }^{\mathrm{TM}}$ in 48 patients with difficult airways by Liu et al, it was observed that though all patients could be ventilated, optimum ventilation without gas leak or high inspiratory pressure was achieved only in 46 patients $(95.8 \%) .^{18}$ In $47.9 \%$ patients, the larynx was visualized immediately while in $47.9 \%$ manipulations were required to view the larynx. Intubation could be performed in $95.8 \%$ of patients. Sreevathsa and associates compared fibreoptic-guided intubating laryngeal mask airway (ILMA) and LMA CTrach ${ }^{\mathrm{TM}}$ and observed that although the insertion time for the two devices was similar, time taken to intubate the trachea was significantly shorter with LMA CTrach ${ }^{\mathrm{TM}}$ as compared with the ILMA-FOS. ${ }^{19}$

Epiglottic downfolding is the commonest cause of suboptimal ventilation and obstruction to the passage of endotracheal tube through LMA Fastrach ${ }^{\mathrm{TM}}$ and poor initial view is responsible for delayed or failed intubation through the LMA $\mathrm{CTrach}^{\mathrm{TM}}$. Several manoeuvres are recommended to overcome this difficulty. Keller and colleagues observed that the incidence of epiglottic down-folding after LMA Fastrach ${ }^{\mathrm{TM}}$ insertion may be as high as $80 \% .{ }^{20}$ In our study 21 patients in group CT and 28 patients in group FT required various manoeuvres for optimal ventilation, view and tracheal intubation. The commonest being up-down manoeuvre required in 13 patients in group CT and 14 patients in group FT $(p=0.154)$. Followed by partial withdrawal required in 6 patients in both groups $(p=0.154)$ and complete withdrawal was required in 2 patients in group CT as compared to 8 patients in group FT $(p=0.154)$. In addition to epiglottic downfolding, poor initial views have also been reported due to fogging of the lens or secretions and lubricating jelly covering the lens with LMA CTrach ${ }^{\mathrm{TM}}$. Similar difficulty was encountered in the present study. In 2 patients the CTrach had to be removed and reinserted after cleaning. This led to prolongation of intubation time. Deterioration of the fibreoptic bundles of the LMA CTrach ${ }^{\mathrm{TM}}$ due to repeated sterilization is also responsible for poor view.

In our study, 2 patients in group CT and 7 patients in group FT had second attempt tracheal intubation $(p=0.154)$ and 1 patient in group FT had third attempt tracheal intubation. Multiple blind attempts with the LMA Fastrach ${ }^{\mathrm{TM}}$ may traumatize the airway. Forceful attempts may even cause arytenoid dislocation. Provided the larynx can be seen with the LMA CTrach ${ }^{\mathrm{TM}}$, the first-attempt intubation success rate is very high. Minor adjustments can be made while viewing the insertion of the endotracheal tube, to prevent impingement of the tube on the arytenoids or vocal cords. ${ }^{8}$ Although optimization of the LMA CTrach ${ }^{\mathrm{TM}}$ placement and view takes time, oxygenation was maintained throughout the intubation procedure.

In our study blood on the airway device was seen in one patient of Group CT as compared to two patients in Group FT $(p=1.00)$. Sore throat was reported by two patients of Group CT as compared to four patients of Group FT ( $p=0.678)$. Abdi and co-workers reported pulmonary aspiration that occurred during tracheal intubation with the LMA CTrach ${ }^{\mathrm{TM}}$ in a male morbidly obese patient admitted for elective gastric banding. ${ }^{21}$ They suggested that the manipulations of the LMA CTrach $^{\mathrm{TM}}$ such as up-down manoeuvre may lead to pulmonary aspiration in the case of regurgitation of gastric content. There was no incidence of pulmonary aspiration in our study.

The LMA CTrach ${ }^{\mathrm{TM}}$ system is portable, can easily be handled by a single operator, and requires less preparation time than a fibreoptic bronchoscope. An important limitation of LMA CTrach $^{\mathrm{TM}}$ reported in the literature is the failure to view the larynx due to secretions. An innovative method of cleaning the fibreoptic tips with a swab inserted through the LMA CTrach $^{\mathrm{TM}}$, without having to remove the LMA CTrach ${ }^{\mathrm{TM}}$ was reported by Maurtua and colleagues. ${ }^{22}$ Although, the LMA CTrach $^{\mathrm{TM}}$ is cheaper than a fibreoptic bronchoscope, it is much more expensive than the LMA Fastrach ${ }^{\mathrm{TM}}$ and therefore its cost affectivity needs to be analysed.

Our study has few limitations. First, all patients had Mallampatti class 1 and 2 and Cormack and Lehane grade I and II on laryngoscopy. The BMI of our patients was normal, and there were a high proportion of female patients. Secondly, we did not use fibreoptic bronchoscopy to diagnose the cause of failed intubation attempts with the LMA Fastrach ${ }^{\mathrm{TM}}$. Thirdly, we used muscle relaxants in all patients. Although we checked that it was possible to ventilate the lungs with facemask, we used relaxants because they may reduce complications, particularly during insertion of ETT and removal of LMA over the ETT. Fourthly, it was impossible to blind the investigators to the system they were using.

To conclude, both airway devices namely LMA Fastrach ${ }^{\mathrm{TM}}$ or LMA CTrach ${ }^{\mathrm{TM}}$ appears comparable and efficacious and the overall success of tracheal intubation.

\section{REFERENCES:}

1. Brain AJ, Verghese C, Addy EV, Kapila A. The intubating laryngeal mask: I. Development of a new device for intubation of the trachea. Br J Anaesth 1997; 79:699-703.

2. Brain $A J$. The laryngeal mask airway: a new concept in airway management. BrJAnaesth 1983;55:801-7.

3. Practice guidelines for management of the difficult airway: An updated report by the American Society of Anesthesiologists Task Force on Management of the Difficult Airway. Anesthesiology 2003;98:1269-77.

4. Ferson D. LMA. What's old - What's new: Patients with difficult airway. ASA Annual meeting refresher courses lectures. Las Vegas. 2003;218: 1-6.

5. Brain AJ.LMA-Fastrach Instruction Manual, 1997.

6. Brain AJ, Verghese C, Addy EV, Kapila A, Brimacombe J. The intubating laryngeal mask. II. a preliminary clinical report of a new means of intubating the trachea.BrJAnaesth 1997;79:704-9.

7. Ferson DZ, Rosenblatt WH, Johansen MJ, Osborn I, Ovassapian A. Use of the intubating LMA-Fastrach ${ }^{\mathrm{TM}}$ in 254 patients with difficult-to-manage airways. Anesthesiology 2001;95:1175-81.

8. Eugene, Goy, Raymond, Lim, Yvonne M, Chen, Fun-Gee. Success of Tracheal Intubation with Intubating Laryngeal Mask Airways: A Randomized Trial of the LMA FastrachTM and LMA CTrachTM.Anesthesiology 2008; 108(4):621-626.

9. LMA CTrachTM Instruction Manual. Henley on Thames, United Kingdom, LMA International Services Limited, 2005.

10. Liu EH, Goy RW, Chen FG. The LMA CTrachTM, a new laryngeal mask airway for endotracheal intubation under vision: Evaluation in 100 patients. Br J Anaesth 2006;96:396-400.

11. Timmermann A, Russo S, Graf B M. Evaluation of LMA CTrachTM - an intubating LMA with integrated fibreoptic system. BrJAneasth 2006;96:516-521. 
12. Baskett PJ, Parr MJ, Nolan JP: The intubating laryngeal mask: Results of a multicentre trial with experience of 500 cases. Anaesthesia 1998;53:1174-1179.

13. Kapila A, Addy EV, Verghese C, Brain AJ. The intubating laryngeal mask airway:An initial assessment of performance. Br JAnaesth 1997;79:710-13.

14. Avidan MS, Harvey A, Chitkara N, et al. The intubating laryngeal mask airway compared with direct laryngoscopy. Br JAnaesth 1999;83:615-7.

15. Goldman $A J$,Wender R, RosenblattWH,Theil D. The fibreoptic intubating LMA CTrachTM: An initial device evaluation (letter). Anesth Analg 2006; 103:508.

16. Dhonneur G, Ndoko SK, Yavchitz A, Foucrier A, Fessenmeyer C, Pollian C, Combes X, Tual L. Tracheal intubation with the LMA CTrachTM or Direct laryngoscopy (letter). Anesth Analg 2007; 104(1):227.

17. Dhonneur G, Ndoko SK, Yavchitz A, Foucrier A, Fessenmeyer C, Pollian C Combes X, Tual L. Tracheal intubation with the LMA CTrachTM: need for cricoid pressure. Br J Anaesth 2007;99(6):749-50.

18. Liu EH, Wender R, Goldman AJ. The LMA CTrachTM in patients with difficult airways. Anesthesiology 2009;110:941-43.

19. Sreevathsa S, Nathan PL, John B, Danha RF, Mendonca C: Comparison of fibreoptic-guided intubation through ILMA versus intubation through LMA CTrachTM. Anaesthesia 2008;63:734-7.

20. Keller C,Brimacombe J, Puhringer F. A fibreoptic scoring system to assess the position of the laryngeal mask airway devices. Interobserver variability and a comparison between the standard, flexible and intubating laryngeal mask a comparison between the standard, flexible and intubating laryngeal mask
airways. Anasthesiol Intensivmed Notfallmed Schmerzther 2000;35:692-294.

21. AbdiW,Ndoko S, Amathieu R, Dhonneur G. Evidence of pulmonary aspiration during difficult airway management of a morbidly obese patient with the LMA CTrachTM.Br J Anaesth 2008; 100(2):275-277.

22. Maurtua MA, Maurtua DB, Zura A, Doyle DJ: Improving intubation success using the CTrach ${ }^{\text {TM }}$ laryngeal mask airway (letter). Anesthesiology 2007; 106:640-1. 